\title{
On the Capacities of Spectrum-Sharing Systems with Transmit Diversity
}

\author{
Asaduzzaman $\cdot$ Hyung-Yun Kong
}

\begin{abstract}
Motivated by recent works on spectrum-sharing systems, this paper investigates the effects of transmit diversity on the peak interference power limited cognitive radio(CR) networks. In particular, we derive the ergodic and outage capacities of a spectrum-sharing system with multiple transmit-antennas. To derive the capacities, peak interference power constraint is imposed to protect the primary receiver. In a CR transmitter and receiver pair with multiple antennas at the transmitter side, the allowable transmit power is distributed over the transmit-antennas to achieve transmit diversity at the receiver. We investigate the effect of this power distribution when a peak interference power constraint is imposed to protect the primary receiver. We show that the transmit diversity does not improve the ergodic capacity compared to the single-antenna system. On the other hand, the transmit diversity significantly improves the outage capacity. For example, two transmit-antennas improve the outage capacity 10 times compared to the singleantenna with a $0 \mathrm{~dB}$ interference constraint.
\end{abstract}

Key words : Cognitive Radio, Spectrum-Sharing, Ergodic Capacity, Transmit Diversity.

\section{Introduction}

In recent years, the demand for radio spectrum has rapidly increased due to the dramatic growth of wireless applications. In current spectrum management policy, most of the spectrum has already been allocated and it is difficult to find aditional spectrum for the new wireless applications. As a solution of this problem, the concept of cognitive radio(CR) has been proposed in [1]. In cognitive radio networks, unlicensed $\mathrm{CR}$ users access to the frequency band of a licensed network(primary network) opportunistically using its cognition capabilities.

In CR networks, CR users may coexist with the primary users either on a non-interference basis or an interference-tolerant basis. In case of non-interference basis systems, the CR users are allowed to operate in the unused frequency bands, commonly known as spectrum holes or white spaces ${ }^{[1]}$. Contrarily, interference tolerant basis systems allow CR users to access the frequency band of the primary users provided that the interference power level at the primary-receivers is kept below some certain threshold ${ }^{[2],[3]}$. Such limits on the interference power is commonly known as interference temperature.

In such interference temperature based model, it is assumed that the primary users are capable of mitigating interference if it is within the predefined interference threshold. Hence, the proposed interference tolerant basis model can be used in any scenario in which the primary users are willing to handle small amounts of interfe- rence. From the primary users' point of view, the secondary access does not affect its operation as long as the total interference power at the primary-receiver remains below the interference threshold. Therefore, the power emitted from a secondary transmitter does not have to be limited as long as the interference inflicted on the primary-receiver is below the threshold.

In this paper, we consider a spectrum-sharing scenario where the CR users access the frequency band of the primary users provided the interference power level at the primary-receivers is kept below some certain threshold. The secondary users use their cognitive capabilities to decide the level of transmit power in order to protect the primary-receiver. In such scenarios, the main objective of a CR transmitter-receiver pair is to maximize the link capacity by maintaining the interference temperature constraints. The ergodic and outage capacities and the power allocation problems of such networks for single-antenna fading channel have been investigated in [2] and [3]. In this paper, we analyzed the effect of transmit diversity on the capacities of the secondary network.

It is well known that transmit diversity is an effective way to mitigate channel fading with a transmit power constraint $^{[4]}$. In interference limited CR networks, the users may need to operate in low signal to noise ratio(SNR) to protect the primary-receivers. Hence, the capacities of such interference limited systems with transmit diversity are important. We derive the ergodic and outage capacities of an interference limited CR network with multiple

Manuscript received June 29, 2010 ; revised September 6, 2010. (ID No. 20100629-021J)

Department of Electrical Engineering, University of Ulsan, Ulsan, Korea.

Corresponding Author : Hyung-yun Kong (e-mail : hkong@mail.ulsan.ac.kr) 
transmit-antennas and a single receive-antenna. Interestingly, the ergodic capacity of the multi-antenna system is lower than the single-antenna system for all interference temperature. On the other hand, the outage capacity of the multi-antenna system improves significantly.

\section{II . System Models}

We consider a spectrum-sharing scenario where the secondary-users coexist with the primary-users and they use the same frequency band. Assume that the secondary transmitters are equipped with $\mathrm{M}$ antennas. This work focuses on the effect of the transmit diversity on the interference temperature constraint $\mathrm{CR}$ network. To separate the transmit diversity from the received diversity we consider a single received antenna. Consequently, we assume both primary and secondary-receivers are single-antenna devices for simplicity. Therefore, the assumed network model is a simple multi-input singleoutput(MISO) channel. We also assume that a suitable space time block code(STBC) is used by the transmitter. We consider a flat Rayleigh fading channel with AWGN. We assume coherent detection at the receivers and thus only the channel power gains are of interest. Let, $G=\left[g_{1}, g_{2}, \ldots, g_{M}\right]$ and $H=\left[h_{1}, h_{2}, \ldots, h_{M}\right]$ represent the instantaneous channel gains from the secondary transmitter to the secondary and primary-receivers, respectively. For Rayleigh fading, $g_{m}$ and $h_{m}$ are the exponential random variables with hazard rates $\lambda_{g}$ and $\lambda_{k}$, respectively.

The interference power received at the primary-receiver due to the transmission of the secondary transmitter can be given as, $\sum_{m=1}^{M} P_{m} h_{m}$; where $P_{m}$ is the transmit power of antenna $m$. The optimal power allocation for single-antenna CR network has been investigated in [2]. To allocate optimal power over the antennas conditioned on the fading statistics required solving tedious optimization problems. For the sake of simplicity, we consider equal power allocation for all antennas as, $P_{1}=P_{2}=$ $\ldots=P_{M}=P(G, H) / M$. Throughout this paper, we only consider the allocation of total transmit power, $P(G, H)$.

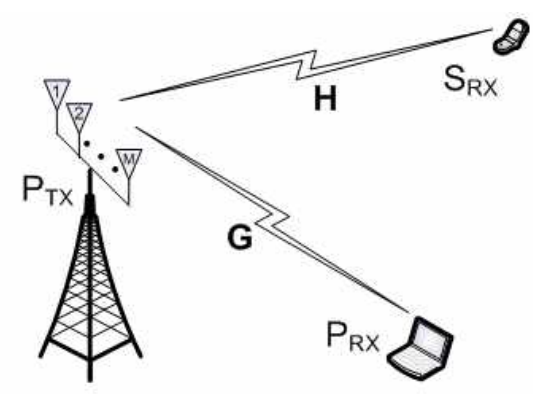

Fig. 1. System model.
The transmit power constraint in terms of the peak interference power $Q$ can be given as

$$
\frac{P(G H)}{M} \sum_{m=1}^{M} h_{m}=\frac{P(G H)}{M} h_{T} \leq Q
$$

where $h_{T}=\sum_{m=1}^{M} h_{m}$. Now the received SNR at the secondary-receiver can be written as,

$$
\gamma \leq \frac{P(G, H)}{M N_{0}} \sum_{m=1}^{M} g_{m}=\frac{P(G, H)}{M N_{0}} g_{T}
$$

where $N_{0}$ is the AWGN variance and $g_{T}=\sum_{m=1}^{M} g_{m}$. Since, $g_{m}$ and $h_{m}$ are the exponential random variables, $g_{T}$ and $h_{T}$ are random variables of the Chi-square distribution with $2 \mathrm{M}$ degrees of freedom [5].

\section{2-1 Capacities}

In this section, we derive the ergodic and outage capacities of a MISO based CR network. For both cases, we consider a Rayleigh fading environment with a received power constraint on a third party's receiver (primary-receiver), as given in (1).

\section{2-2 Ergodic Capacity}

Ergodic capacity is the long term average achievable rate constraint on the power but on the delay. Assuming $g_{m}$ and $h_{m}$ are independent of each other for all $m$, the ergodic capacity of the system can be given as

$$
\begin{aligned}
& C_{e r}=\max E_{G, H}\left\{r B \log \left(1+\frac{g_{T} P(G, H)}{N_{0} B}\right)\right\} \\
& \text { s.t } h_{T} P(G, H) \leq Q_{p k}
\end{aligned}
$$

where $r$ is the capacity loss of the STBC codes and $E_{G, H}$ represents the expectation over the joint probability density function(pdf) of $G$ and $H$. Using the peak interference power constraint and the received SNR of (1) and (2) we can easily calculate the ergodic capacity as

$$
\begin{aligned}
C_{e r} & =E_{g_{T}, h_{T}}\left\{r B \log \left(1+\frac{Q_{p k}}{N_{0} B} \frac{g_{T}}{h_{T}}\right)\right\} \\
& =\int_{0}^{\infty} r B \log \left(1+\frac{Q_{p k}}{N_{0} B} \frac{g_{T}}{h_{T}}\right) f_{g_{T} / h_{T}}(x) d x
\end{aligned}
$$

where $f_{g_{T} / h_{T}}(x)$ is the pdf of the random variable $g_{T} / h_{T}$ which is derived in the Appendix.

The ergodic capacity of (4) against the peak interference power for different numbers of antennas is shown in Fig. 2. Results indicate that the ergodic capacity of the CR network decreases as the number of antennas increases. This result is quite interesting because the diversity gain achieved by the multiple transmit-antennas 


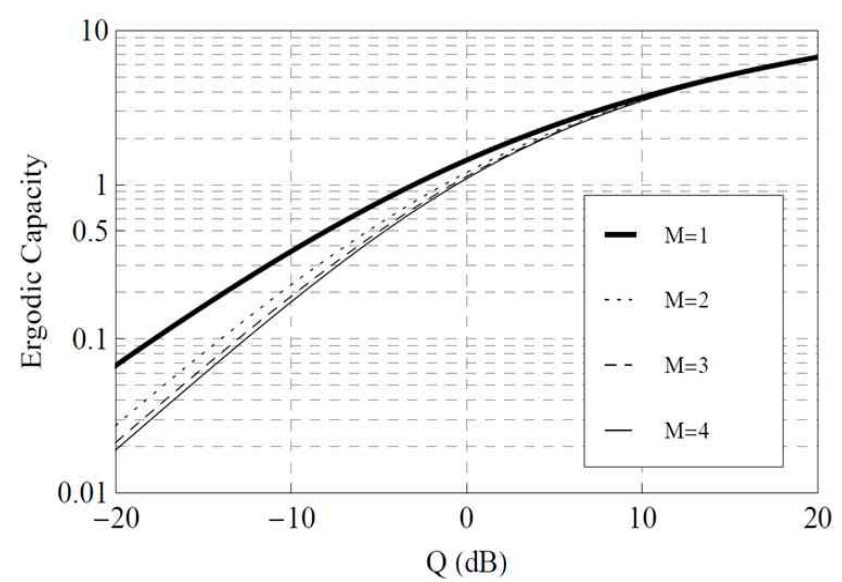

Fig. 2. Ergodic capacity with peak received-power constraint.

is not useful for interference temperature-limited CR systems. Because the interference power received by the primary-receiver also increases as the number of antennas increasee along with the increased received SNR at the CR-receiver.

\section{2-3 Outage Capacity}

The ergodic capacity is the measure of the maximum achievable long-term rate without considering any delay constraint. However, in reality, many wireless applications have certain delay constraints. In such scenarios, delay limited capacity, defined as the constant rate that is achievable in all fading sates, is more suitable. However, in Rayleigh fading the delay limited capacity is zero for any finite average power limitation. Alternatively, outage capacity that exist a capacity-versus-outage phenomenon, is a good measure of the channel capacity for Rayleigh fading channels ${ }^{[5]}$. In this sub-section, we will determine the outage capacity with truncated channel inversion ${ }^{[6]}$ under a peak interference constraint at the primary-receiver.

For outage capacity with peak interference temperature constraint, the power allocation policy can be written using the well known truncated channel inversion power control $^{[6]}$ as

$$
P(G, H)=\left\{\begin{array}{l}
\frac{\alpha}{g_{T}}, \frac{g_{T}}{h_{T}} \geq c \\
0, \quad \text { otherwise }
\end{array}\right.
$$

where $\alpha$ and $c$ are non-negative constants and can be determined from the peak interference power constraint and the outage probability $\operatorname{Pr}\left[\frac{g_{T}}{h_{T}} \leq c\right]$. Using the fact that the transmission is abandoned when $\frac{g_{T}}{h_{T}}$ is less than $c$, the outage probability can be found as

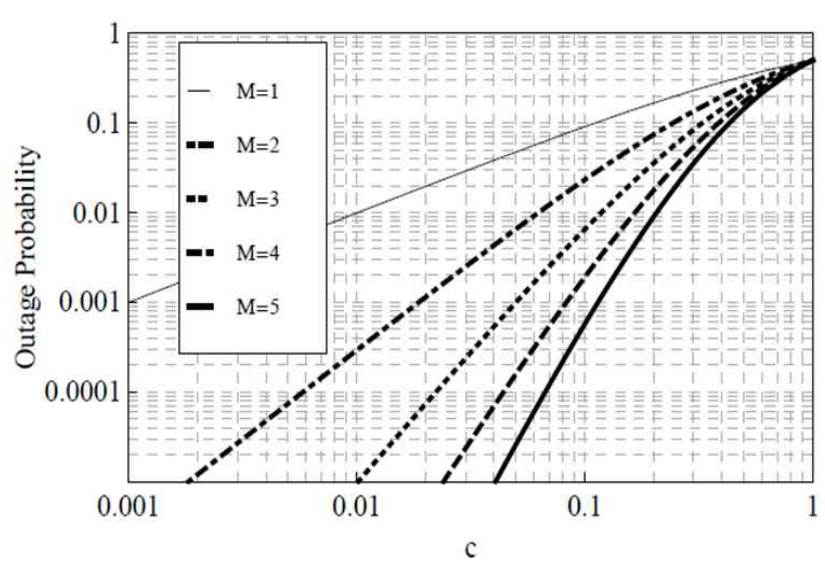

Fig. 3. Outage probability for different numbers of antennas with peak received-power constraint.

$$
\begin{aligned}
P_{\text {OUT }} & =\operatorname{Pr}\left[\frac{g_{T}}{h_{T}} \leq c\right] \\
& =F_{g_{T} / h_{T}}(c)
\end{aligned}
$$

where $F_{g_{T} / h_{T}}$ is the cumulative distribution function(CDF) of the random variable $\frac{g_{T}}{h_{T}}$ which is derived in the Appendix.

Fig. 3 shows the outage probability for different numbers of transmit-antennas(M). Clearly, the outage probability decrease as the number of transmit-antennas increases for $c<1$.

To satisfy the peak interference power constraint the inequality $\alpha \leq c Q$ must be satisfied. Now the outage capacity under the truncated channel inversion power control can be given as

$$
\begin{aligned}
& C_{e r}=r B \log \left(1+\frac{\alpha}{N_{0} B}\right) \\
& \leq r B \log \left(1+\frac{c Q}{N_{0} B}\right)
\end{aligned}
$$

where, the value of $c$ is the solution of (6)

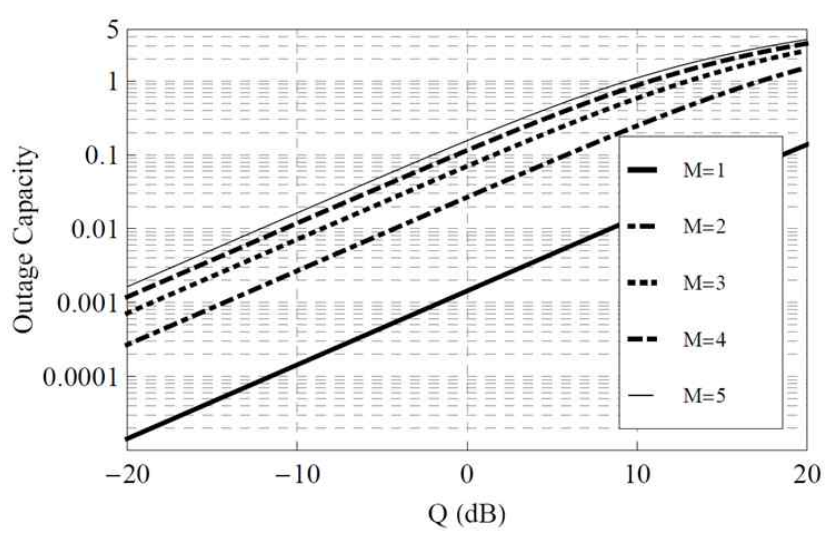

Fig. 4. Outage capacity with peak received-power constraint for $P_{\text {OUT }}=0.001$. 
The outage capacity of (7), for different numbers of transmit-antennas is plotted against the peak interference constraint in Fig. 4. In this figure, we consider an outage probability of $P_{\text {OUT }}=0.001$. Unlike the ergodic capacity, the outage capacity improves as the number of antennas increases. Considering that the system achieves transmit diversity, a smaller transmit power is needed to achieve the target outage probability which leads to reduced interference at the primary-receiver. Equivalently, for certain peak interference-power, the system with transmitdiversity operates on a small outage probability. Hence, the outage capacity of the system improves.

\section{Conclusion}

In this paper, we investigate the information-theoretic limits on the interference temperature based CR MISO channel. The ergodic and the outage capacities of such systems are derived. We also derive the closed form expression of the outage probability of the CR network. We show that the transmit diversity does not improve the ergodic capacity, but improves the outage capacity significantly. Numerical results are given to support the arguments. Results show that the outage capacity of a CR system with only two antennas is 10 times higher than the single-antenna system.

\section{Appendix}

For Rayleigh fading, $g_{m}$ and $h_{m}$, for all $m$, are the exponential random variables with hazard rates $\lambda_{g}$ and $\lambda_{h}$, respectively. Hence, $g_{T}=\sum_{m=1}^{M} g_{m}$ and $h_{T}=\sum_{m=1}^{M} h_{m}$ are the random variables with the Chi-square distribution with $2 \mathrm{M}$ degrees of freedom with pdf [4, Eq.9.5]

$$
\begin{aligned}
& f_{g_{T}}(x)=\frac{\lambda_{g}}{(M-1) !} x^{M-1} e^{-\lambda_{g} x} \\
& f_{h_{T}}(x)=\frac{\lambda_{h}}{(M-1) !} y^{M-1} e^{-\lambda_{k} y}
\end{aligned}
$$

Now the pdf of the random variable $\frac{g_{T}}{h_{T}}$ can be written as [7]

$$
\begin{aligned}
f_{g_{T} / h_{T}}(z) & =\int_{y=0}^{\infty} y f_{g_{T}}(y z) f_{h_{T}}(y) d y \\
& =\frac{\lambda_{g} \lambda_{h}}{\{(M-1) !\}^{2}} \int_{y=0}^{\infty} y^{2 M-1} z^{M-1} e^{-\left(\lambda_{g} z+\lambda_{h}\right)} d y \\
& =\frac{\lambda_{g} \lambda_{h} \Gamma(2 M)}{\{(M-1) !\}^{2}} z^{M-1}\left(\lambda_{g} z+\lambda_{h}\right)^{-2 M}
\end{aligned}
$$

where, $\Gamma($.$) is the Gamma function. Now, the CDF for$ $\frac{g_{T}}{h_{T}}$ can be written as

$$
\begin{aligned}
& F_{g_{T} / h_{T}}(z)=\int_{0}^{z} f_{g_{T} / h_{T}}(z) d z \\
& =\frac{\lambda_{g} \lambda_{h} \Gamma(2 M)}{\{(M-1) !\}^{2}} \frac{z^{L}}{L \lambda_{h}^{2 L}}{ }_{2} F_{1}\left(L, 2 L ; L+1 ; \frac{\lambda_{g}}{\lambda_{y}} z\right)
\end{aligned}
$$

where, ${ }_{2} F_{1}(., . ;, .$.$) is the Gauss's hyper geometric function$ determined in [8].

This research was supported by Basic Science Research Program through the National Research Foundation of Korea(NRF) funded by the Ministry of Education, Science and Technology(No. 2009-0073895).

\section{References}

[1] J. Mitola, III, G. Q. Maguire, Jr., "Cognitive radio: making software radios more personal", Personal Communications IEEE, vol. 6, pp. 13-18, 1999.

[2] X. Kang, et al., "Optimal power allocation for fading channels in cognitive radio networks: Ergodic capacity and outage capacity", Wireless Communications, IEEE Transactions on, vol. 8, pp. 940-950, 2009.

[3] L. Musavian, S. Aissa, "Ergodic and outage capacities of spectrum-sharing systems in fading channels", in Global Telecommunications Conference, 2007. GLOBECOM '07. IEEE, pp. 3327-3331, 2007.

[4] J. H. Winters, "The diversity gain of transmit diversity in wireless systems with Rayleigh fading", Vehicular Technology, IEEE Transactions on, vol. 47, pp. 119-123, 1998.

[5] E. Biglieri, et al., "Fading channels: informationtheoretic and communications aspects", Information Theory, IEEE Transactions on, vol. 44, pp. 26192692, 1998.

[6] A. J. Goldsmith, P. P. Varaiya, "Capacity of fading channels with channel side information", Information Theory, IEEE Transactions on, vol. 43, pp. 19861992, 1997.

[7] A. Papoulis, S. U. Pillai, Probability, Random Variables, and Stochastic Processes, 4th Ed., Boston: McGraw-Hill, 2002.

[8] M. Abramowitz, et al.(1972, Handbook of mathematical functions with formulas, graphs, and mathematical tables(10th printing, with corrections, Ed.). 


\section{Asaduzzaman}

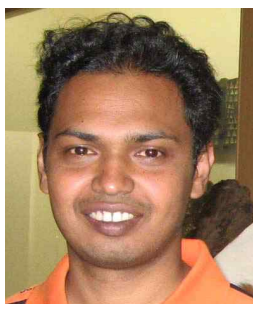

received the B. Sc. Engineering Degree in electrical and electronics engineering in 2001 from Chittagong University of Engineering and Technology, Bangladesh. From 2001 to 2005 he was a faculty member at the same university. He is currently working toward the Ph.D. degree in the Department of Electrical Engineering, University of Ulsan, Korea. His major research interests include wireless communication systems with emphasis on cooperative communications and MIMO systems, wireless sensor networks, modulation and coding techniques.

\section{Hyung Yun Kong}

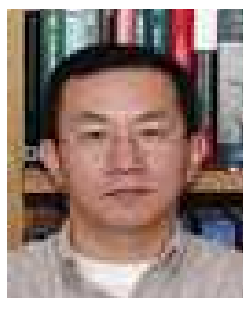

received the M.E. and Ph.D. degrees in electrical engineering from Polytechnic University, Brooklyn, New York, USA, in 1991 and 1996, respectively, He received a BE in electrical engineering from New York Institute of Technology, New York, in 1989. Since 1996, he has been with LG Electronics Co., Ltd., in the multimedia research lab developing PCS mobile phone systems, and from 1997 the LG chairman's office planning future satellite communication systems. Currently he is a Professor in electrical engineering at the University of Ulsan, Korea. His research area includes channel coding, detection and estimation, cooperative communications, cognitive radio and sensor networks. He is a member of IEEK, KICS, KIPS, IEEE, and IEICE. 